\title{
Closed-suction drainage in thoracolumbar spinal surgery-clinical routine without evidence? a systematic review
}

\author{
Klaus John Schnake ${ }^{1,2} \cdot$ Matthias Pumberger ${ }^{3}$. Denis Rappert ${ }^{1} \cdot$ Achim Götz $^{1}$ - Oleksandr Zolotoverkh ${ }^{1}$ \\ Rita Waligora ${ }^{3} \cdot$ Max Joseph Scheyerer ${ }^{4}$. Spine Section of the German Society of Orthopaedics and Trauma
}

Received: 11 January 2021 / Revised: 26 November 2021 / Accepted: 27 November 2021 / Published online: 29 January 2022

(c) The Author(s) 2022

\begin{abstract}
Purpose The considered benefit of surgical drain use after spinal surgery is to prevent local accumulation of a haematoma by decompressing the closed space in the approach of the surgical site. In this context, the aim of the present systematic review was to prove the benefit of the routine use of closed-suction drains.

Methods We conducted a comprehensive systematic review of the literature according to the Preferred reporting items for systematic reviews and meta-analyses (PRISMA) checklist and algorithm.

Results Following the literature search, 401 potentially eligible investigations were identified. Eventually, a total of 24 studies with 8579 participants were included. Negative suction drainage led to a significantly higher volume of drainage fluid. Drainage duration longer than $72 \mathrm{~h}$ may be associated with a higher incidence of Surgical side infections (SSI); however, accompanying antibiotic treatment is unnecessary. Regarding postoperative haematoma and neurological complications, no evidence exists concerning their prevention. Hospital stay length and related costs may be elevated in patients with drainage but appear to depend on surgery type.

Conclusions With regard to the existing literature, the use of closed-suction drainage in elective thoracolumbar spinal surgery is not associated with any proven benefit for patients and cannot decrease postoperative complications.
\end{abstract}

Keywords Wound drains $\cdot$ Postoperative haematoma $\cdot$ Complications $\cdot$ Spinal surgery

\section{Introduction}

Currently, spinal surgery provides a recognised treatment option for various pathologies starting with degenerative changes, fractures and deformities up to tumour [1-3]. The considered benefit of surgical drain use in those cases is to prevent local accumulation of a haematoma by decompressing the closed space in the approach of the surgical site $[4,5]$. With the reduction of a haematoma, it is postulated that the possibility of ecchymosis, wound dehiscence, infection and especially neurologic compromise would decrease [6].

Evidence regarding the use of wound drains is poor, and the application is rather generous [7]. In contrast to the

Rita Waligora

Rita.Waligora@charite.de

1 Center for Spine and Scoliosis Therapy, Malteser Waldkrankenhaus St.,Marien, Erlangen, Germany

2 Department of Orthopedics and Traumatology, Paracelsus Private Medical University Nuremberg, Nuremberg, Germany

3 Spine Department, Center for Musculoskeletal Surgery, Charité University Medicine Berlin, Berlin, Germany

4 Department of Orthopedic and Trauma Surgery, Medical Faculty, University of Cologne, Kerpener Straße 62, 50937 Cologne, Germany 
aforementioned advantages, some authors postulate that a drain carries the risk of deep wound contamination and higher rates of infection due to the direct connection with the outside environment [5, 8-10]. Others assess the use of a postoperative drain following a routine posterior lumbar fusion critically due to its presence as a foreign body and the additional expense of the procedure [11].

While these theories have been tested in many investigations, there is still no definite consensus. The aim of this systematic literature review was to determine recommendations from the current available evidence with respect to the use of drains, application type, localisation and duration. In addition, we illuminate the influence on any reduction in postoperative complications and the effects on blood loss, need for blood products and hospital stay.

\section{Material and methods}

\section{Study design}

We conducted a comprehensive systematic review of the literature according to the Preferred reporting items for systematic reviews and meta-analyses (PRISMA) checklist and algorithm [12].

\section{Study characteristics}

Investigations between 2010 and 2020 were included. For analyses, prospective and retrospective observational investigation studies that dealt with wound drains after thoracic and lumbar spinal surgery were considered. Furthermore, only articles in German or English were involved.

\section{Information sources}

The authors performed an initial search of PubMed and Google Scholar databases for investigations for possible inclusion in the review.

\section{Search}

The keywords used in the research were ('Wound drainage' OR 'drainage' OR 'drain' OR 'negative wound pressure therapy') AND (English OR German) NOT (cervical spine) AND ('Spine surgery' OR ‘spinal surgery' OR ‘spinal fusion' OR ‘spinal decompression') AND (English OR German) NOT (cervical spine).

\section{Study selection}

The authors limited the research to observational studies, while systematic reviews, meta-analyses, case series and case reports were excluded. Titles and abstracts were reviewed. Duplicates were removed and full texts were checked for suitability. In cases where a decision could not be taken based on information from the title and abstract, the full text was evaluated. The final decision was made based on the analysis of the full text. The study selection process was carried out independently by two authors (KJS, MJS).

\section{Data items}

The main prerequisite was that included studies dealt with wound drains after thoracic and lumbar spinal surgery. The authors performed an initial search of both databases for investigations for possible inclusion in the review. First, title and abstracts were screened. In cases where a decision could not be taken due to the information from the title and abstract the full text was evaluated. In the end, the final decision was made based on the analysis of the full text.

Studies were selected according to the following inclusion criteria: (a) negative pressure suction drainage versus overflow drainage; (b) duration of treatment with suction drainage; (c) need for accompanying antibiotic treatment; (d) impact on wound healing; (e) impact on blood loss and transfusion requirements; (f) impact on costs and hospital stay; and (g) impact on postoperative complications (surgical site infection, postoperative fever, haematoma, neurological complications and reoperation).

\section{Synthesis of results}

We extracted data concerning study characteristics including author's name, title, year of publication and journal published. Outcome parameters were analysed according to the above-mentioned inclusion criteria. For all included studies, we used the Oxford Centre for Evidence-Based Medicine (2011) guidelines for defining the level of evidence [13]. The strength of recommendation was defined using the GRADE approach (Grading of recommendations assessment, development and evaluation). Regarding the risk of bias every included investigation was graded with respect to the Newcastle Ottawa scale.

\section{Results}

\section{Study characteristics}

The initial research identified 401 potentially eligible investigations in both databases. Amongst these, 364 were excluded after screening the title and abstract as they did not meet the inclusion criteria. After screening the 
Fig. 1 PRISMA flowchart

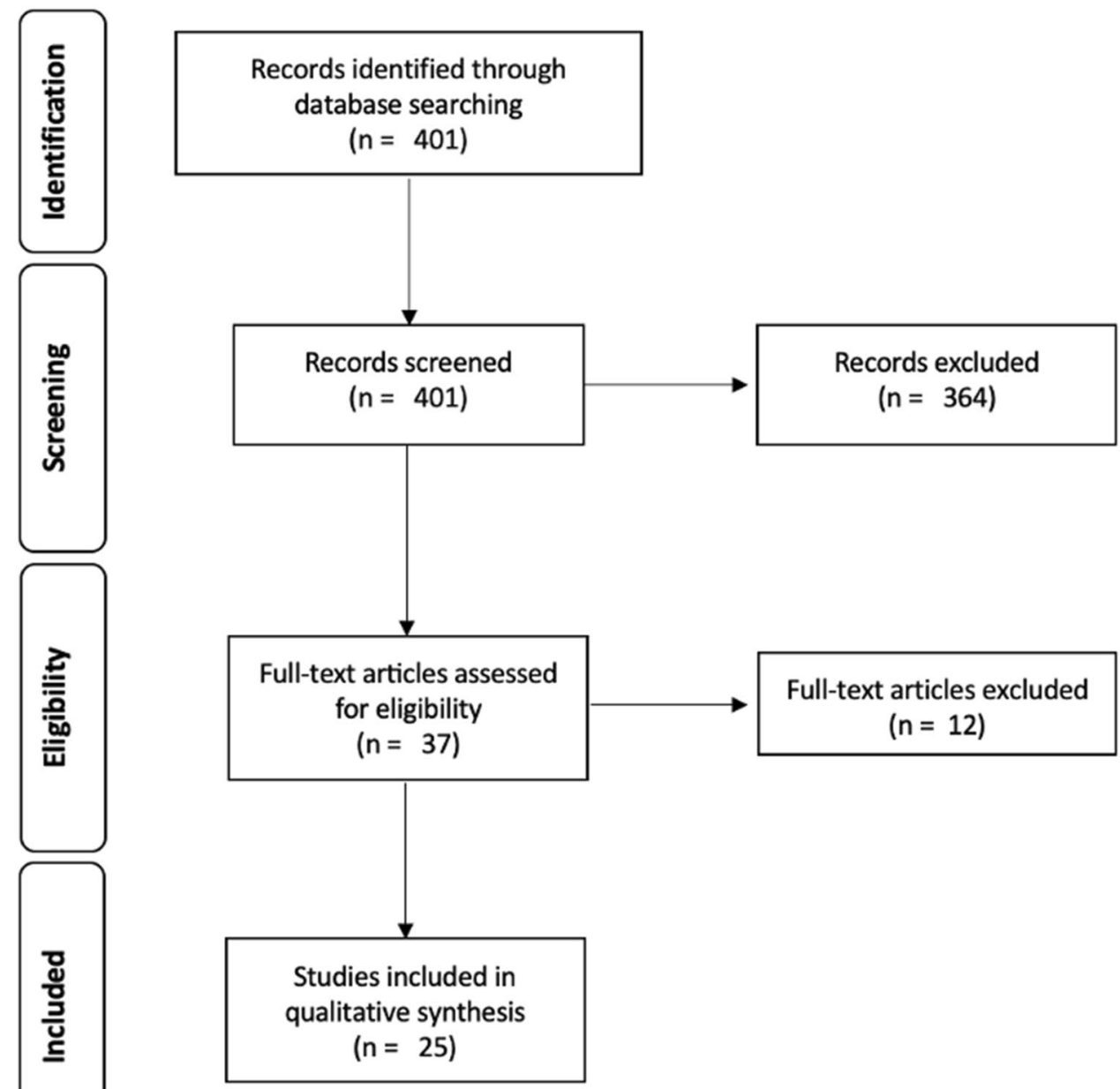

abstracts and full texts further, 12 were excluded for the same reason. Therefore, 25 studies were finally included in the systematic review and were analysed. The full selection process is shown in the PRISMA flowchart in Fig. 1.

In summary, 8579 participants were analysed within this systematic review. All investigations were published in the English language and had a cohort design. Seventeen were retrospective investigations and 18 were prospective. Table 1 shows the study characteristics of the included investigations, including the level of evidence.

\section{Negative pressure suction drainage versus overflow drainage}

Chen et al. retrospectively compared the clinical outcomes between natural pressure drainage and negative pressure drainage after posterior lumbar interbody fusion in 132 patients [14]. The median drainage volume on the first postoperative day and the total volume of postoperative drainage was significantly higher in the negative pressure group (both $P=0.000$ ). The median total drainage days between the natural pressure and negative pressure groups were $2.93 \pm 0.55$ vs. $3.51 \pm 0.71$ days $(p=0.0001)$. No difference in patient characteristics, operative data, postoperative temperature or complications were reported.

\section{Continuous negative pressure drainage versus intermittent-clamped drainage}

Hao et al. prospectively compared the usage of Continuous negative pressure drainage (CNPD) with Intermittentclamped drainage (ICD) after lower lumbar decompression and internal fixation [15]. Overall, 156 patients were randomly assigned to both groups. The mean total drainage amount of the single-segment and two-segment procedures was significantly greater in the CNPD group than the ICD group $(p<0.01)$. No significant differences were found regarding postoperative temperature, wound problems or complications between groups. The authors concluded that 
Table 1 Study characteristics of the included investigations

\begin{tabular}{|c|c|c|c|c|c|c|}
\hline & Year & Study design & Sample size (n) & $\begin{array}{l}\text { Level of } \\
\text { evidence }\end{array}$ & $\begin{array}{l}\text { Strength of recom- } \\
\text { mendation }\end{array}$ & Newcastle-Ottawa scale \\
\hline Chen et al. [14] & 2020 & Retrospective & 132 & 3 & Moderate & Good quality \\
\hline Scuderi et al. [6] & 2005 & Retrospective & 85 & 3 & Low & Poor quality \\
\hline Kanayama et al. [31] & 2010 & Retrospective & 560 & 3 & Moderate & Fair quality \\
\hline Kochai et al. [32] & 2019 & Retrospective & 63 & 3 & Low & Fair quality \\
\hline Blank et al. [24] & 2003 & Prospective & 30 & 2 & Moderate & Fair quality \\
\hline Ovadia et al. [26] & 2019 & Prospective & 100 & 2 & High & Good quality \\
\hline Liang et al. [16] & 2013 & Prospective & 105 & 1 & High & Good quality \\
\hline Diab et al. [33] & 2012 & Retrospective & 500 & 2 & Moderate & Good quality \\
\hline Urquhrt et al. [22] & 2019 & Prospective & 552 & 1 & High & Good quality \\
\hline Li et al. [28] & 2019 & Retrospective & 448 & 2 & Moderate & Fair quality \\
\hline Pennington et al. [20] & 2019 & Retrospective & 38 & 3 & Low & Fair quality \\
\hline Choi et al. [35] & 2019 & Retrospective & 64 & 3 & Low & Fair quality \\
\hline Gubin et al. [27] & 2019 & Prospective & 161 & 1 & High & Good quality \\
\hline Adogwa et al. [18] & 2018 & Retrospective & 139 & 3 & Moderate & Fair quality \\
\hline Chen et al. [23] & 2018 & Retrospective & 1125 & 3 & Moderate & Good quality \\
\hline Liu et al. [30] & 2018 & Retrospective & 2715 & 4 & Low & Fair quality \\
\hline Lai et al. [29] & 2017 & Retrospective & 26 & 4 & Low & Poor quality \\
\hline Macki et al. [17] & 2017 & Retrospective & 209 & 4 & Moderate & Fair quality \\
\hline Hao et al. [15] & 2016 & Prospective & 156 & 2 & High & Good quality \\
\hline Hung et al. [37] & 2017 & Prospective & 56 & 1 & Moderate & Fair quality \\
\hline Yamada et al. [21] & 2016 & Retrospective & 55 & 4 & Low & Poor quality \\
\hline Ahn et al. [19] & 2015 & Retrospective & 133 & 4 & Low & Poor quality \\
\hline Takemoto et al. [4] & 2015 & Prospective & 314 & 2 & High & Good quality \\
\hline Alsiddiky et al. [25] & 2013 & Retrospective & 411 & 4 & Low & Poor quality \\
\hline Walid et al. [34] & 2012 & Retrospective & 402 & 3 & Moderate & Fair quality \\
\hline
\end{tabular}

ICD is an effective, convenient and safe method for routine use in lower lumbar surgery.

\section{Subfascial versus subcutaneous drainage}

Liang et al. performed a prospective, randomised controlled clinical study to evaluate the efficacy of subcutaneous closed-suction drainage in comparison with conventional closed-suction drainage [16]. A total of 105 patients undergoing posterior instrumented spinal fusion due to adolescent idiopathic scoliosis were randomised into one of the two groups. The mean drainage volume was significantly less in the subcutaneous closed-wound suction drainage group (42 vs. $631 \mathrm{~mL}, p=0.000$ ). However, core temperature values were higher in the subcutaneous closed-wound suction drainage group $(p=0.001)$ as well as fever duration $(p=0.008)$. No differences were found regarding demographic distribution, haemoglobin/haematocrit changes, transfusion requirements or incidence of wound problems (requirements for dressing reinforcement, oozing, subcutaneous haematoma, ecchymosis and infection). The authors concluded that subcutaneous closed-suction drainage offers a reasonable alternative in adolescent idiopathic scoliosis patients undergoing posterior instrumented spinal fusion.

\section{Duration of treatment with suction drainage}

Although drains have commonly been applied for decades in spinal surgery, there is no clear recommendation for the in situ duration. In a retrospective controlled study of 209 patients who underwent posterolateral decompression and fusion for degenerative lumbar spine disease, the median drainage removal interval was on average $36 \mathrm{~h}$ (SD 30-40) from surgery. The authors did not find any significant differences in wound complications and Surgical site infection (SSI) with respect to duration until drain removal [17]. Although time to drain removal was significantly longer in the group where drains were removed in the inpatient setting (pre-discharge cohort) than in the post-discharge cohort (8.28 days vs. 4.65 days, $p<0.001$ ), the incidence of reoperation for SSI did not differ significantly (6.33 vs. $7.69 \%$, $p=0.711)$ [17].

However, the contradictory results regarding a link between duration of suction drainage and SSI in patients 
undergoing elective spinal surgery exist in the literature. In line with the above-mentioned results, Adogwa et al. concluded that the use of drains was not associated with a reduction of SSIs in patients undergoing spinal decompression and fusion [18]. In a retrospective clinical study of 133 patients who underwent primary spinal surgery (37 laminoplasties, 16 decompressions, 80 fusions), there was no significant association between drain tip culture and drainage duration, or between the rate of SSI and drainage duration [19].

However, Pennington et al. showed that early drain removal in patients who underwent surgery for a degenerative condition of the spine decreased the risk of SSI ( $5.5 \mathrm{vs.}$ 3.5 days, $p=0.02$ ) [20].

Similar results were reported by Yamada et al. [21]. In their retrospective study of 1085 primary spinal surgeries and 155 revision cases, the frequency of SSI increased if the drain was removed after more than $72 \mathrm{~h}(p=0.0141)$ [21]. There was no significant difference in the rate of SSI between the cases in which removal was performed within $12-48 \mathrm{~h}$ or more than $48 \mathrm{~h}$ after surgery $(p=0.51)$ [21].

\section{Need for accompanying antibiotic treatment}

Urquhart et al. showed that prolonged postoperative anti-

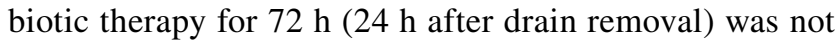
associated with a decrease of SSI after posterior thoracolumbar spinal surgery [22]. In 552 randomised patients, 282 received antibiotic therapy for $24 \mathrm{~h}$ and 270 for $72 \mathrm{~h}$. In the 24-h group, 17 (6.0\%) patients developed a SSI and in the 72-h group 14 (5.2\%) patients did so. Furthermore, the superficial infection rate did not vary in either groups (24-h group: 9.6\% vs. 72-h group: 8.1\%) [22].

The type of tube seems to have no impact on antibiotic therapy and the clinical result. In an investigation by Chen et al., 1125 patients with single-tube drainages and doubletube drainages showed no significant difference. However, the retrospective study design identifying 26 patients with SSIs and no clearly depicted antibiotic regime introduces possible biases in this study [23].

In line with previous studies, a prospective, randomised trial by Takemoto et al. with 314 included patients confirmed that antibiotic necessity was not influenced by the presence of suction drainage. In this study, 170 patients received antibiotic treatment for $24 \mathrm{~h}$ and the other group of 144 patients received antibiotic treatment until drain removal. This trial showed that antibiotic treatment during the whole duration did not decrease the rate of surgical side infections [4]. These findings were confirmed by a retrospective observational study by Yamada et al. [21]. Prophylactic antibiotics were administered during and after surgery, for a total of $48 \mathrm{~h}$, according to the Japanese Orthopaedic Association
Clinical Practice Guidelines on the Prevention of Surgical Site Infections in Bone and Joint.

\section{Impact on wound healing}

The impact of drains on wound healing is controversially discussed in the literature. Blank et al. reported, in a prospective study, the impact of suction drainage and suggested that the use of closed-suction drainage for patients undergoing posterior spinal fusion for adolescent idiopathic scoliosis decreases wound complications [24]. On the other hand, Alsiddiky et al. showed that there was adequate wound healing without using a drain. In this case series, patients were included from 2007 until 2010 who underwent a similar surgical technique for posterior fusion of scoliosis without drain use. There were only two cases $(4,16 \%)$ with superficial skin infection, treated with antibiotics, with full recovery [25]. In addition, there are two further prospective, randomised studies indicating that drains have no impact on wound healing. Ovadia et al. showed that the number of wound healing complications was similar in the study and control groups [26]. Furthermore, Gubin et al. performed an open label randomised control trial with a total of 155 patients. Eighty patients were allocated to a suction drainage group and 75 to a control group. Despite one case of superficial wound inflammation in each group, there were no SSIs [27].

\section{Impact on postoperative complications}

\section{Surgical side infection}

$\mathrm{Li}$ et al. retrospectively investigated the reasons for SSI in 448 consecutive patients with lumbar degenerative disease who underwent classic open transforaminal lumbar interbody fusion [28]. Apart from multifactorial reasons, the use of postoperative drainage $(p=0.004)$ and duration of draining $(p<0.001)$ were significant reasons for SSI. Logistic regression analysis revealed longer time of draining amongst others as being significantly related to SSI $(p<0.05)$ [28].

Similarly, Pennington et al. identified that, amongst other factors, longer drain retention time (5.5 vs. 3.5 days, $p=0.02$ ) was a risk factor for SSI [20].

Furthermore, Liu et al. identified 64 out of 2715 patients and Lai et al. 26 out of 925 patients with SSIs [29]. In both studies, prolonged drainage duration was identified as an independent risk factor $(p=0.016$ and $p<0.05)$ [30].

Nevertheless, contrary results exist. Kananyama et al. reported no SSIs in 560 patients undergoing single-level lumbar decompression or discectomy, whether they had received drains $(n=298)$ or not $(n=262)$ [31]. Similar to results from other investigations on posterior spinal fusion, the risk of SSI was comparable in groups with or without drains $[32,33]$. 
In contrast to the contrary results for SSIs and drainage use, the impact of microbiological drain tip culture is clearer. Two retrospective studies examined the correlation between microbiological drain tip cultures and SSI. Yamada et al. cultured 1240 suction drain tips and revealed 55 positive cases $(4.4 \%)$ [21]. The authors correlated the positive cases with the number of SSIs $(n=18,1.5 \%)$. The sensitivity of drain tip culture for SSI after spinal surgery was calculated as $0 \%$ according to the concordance rate between bacteria isolated in the drain tip culture and SSI cases. There was no correlation between the duration of suction drainage and the drain tip culture results or the onset of SSI within $72 \mathrm{~h}$. Basis on the results, the prognostic value of drain tip culture for SSI was refuted [21].

Ahn et al. reported similar results. They found a positive drain tip culture in 48 of 133 patients $(36.1 \%)$, but only six patients developed a SSI [19]. The sensitivity of drain tip culture for SSI after primary posterior spine surgery was $60.0 \%$, and the specificity was $65.9 \%$. There was no significant association between drain tip culture and duration of drainage just like the rate of SSI and duration of drainage.

\section{Postoperative fever}

Two prospective, randomised controlled studies of posterior spinal fusion in adolescent idiopathic scoliosis reported about postoperative fever. Ovadia et al. included 100 patients with and without drainage [26]. Both groups showed no fever for the first three days but a significantly increased temperature in the no-drain group on day 6 $(p=0.017)$. All other measurements showed no differences between groups. Liang et al. included 105 patients and compared subcutaneous versus conventional closed-wound suction drainage systems [16]. The core temperature was higher $(p=0.001)$ and the duration of fever was longer $(p=0.008)$ in the subcutaneous drainage group. However, the findings were not associated with any disadvantages for the patients.

In a retrospective study with 402 patients who underwent lumbar decompression and fusion, Walid et al. reported a higher amount of fever in the group with drains compared to the group without drains (63.2\% vs. $52.6 \%, p=0.05)$ [34].

\section{Haematoma}

Only two retrospective studies exist regarding postoperative haematoma. Adogwa et al. determined the incidence of postoperative complications after spinal decompression and fusion with and without a subfascial drain [18]. The authors identified $116(83.5 \%)$ patients who had a postoperative drain and $23(16.5 \%)$ who did not. The incidence of postoperative haematoma formation did not differ significantly between groups $(p=0.66)$ [18].

Kananyama et al. reported the results of 560 patients undergoing single-level lumbar decompression or discectomy [31]. The rate of postoperative haematoma was $0.7 \%$ $(2 / 298)$ in the group that received drains and $0 \%(0 / 262)$ in the group that did not $(p>0.5)$.

\section{Neurological complications}

Diab et al. reported the outcomes of a retrospective multicentre study after posterior spinal fusion with instrumentation for adolescent idiopathic scoliosis [33]. In their collective of 500 patients, no differences regarding neural injuries were found between the groups with and without drains $(p>0.1)$ [33].

Alsiddiky et al. performed a case series with 48 paediatric patients with idiopathic scoliosis who underwent posterior spinal instrumentation and fusion without drain use. No neurological complications were noted [25].

\section{Reoperation}

Wound drainage may have an impact on reoperations. However, no studies exist comparing patients with or without drainage regarding this circumstance.

Macki et al. retrospectively reviewed, at a single institution, 209 patients with posterolateral decompression and fusion for degenerative lumbar spine disease [17]. The objective of this study was to determine the incidence and predictors of reoperation for SSI amongst patients where lumbar, closed-wound suction drains were removed in the inpatient setting prior to hospital discharge (pre-discharge cohort, $n=130$ ) versus after inpatient discharge during the first follow-up visit (post-discharge cohort, $n=79$ ). The incidences of reoperation for SSI did not significantly differ (6.33 vs. $7.69 \%$, respectively, $p=0.711$ ) [17].

Scuderi et al. reported the outcomes of 85 patients with posterior lumbar fusions performed by a single surgeon [6]. In this retrospective evaluation, all patients were treated without the use of wound drainage. Two reoperations were necessary $(2.35 \%)$, one due to an infection and one due to a haematoma.

\section{Impact on blood loss and transfusion requirements}

Regarding blood loss depending on the use of drains, type of application, localisation and duration, we refer to the preceding chapters. With a focus on the need for transfusion requirements, nine studies dealt with this question in more detail and came to diverging results [16, 18, 24, 27, 32-36]. 
Choi et al. conducted a retrospective investigation on 50 consecutive patients who had undergone posterior spinal fusion with pedicle screw instrumentation due to Adolescent idiopathic scoliosis (AIS) [35]. After performing multiple linear regression analysis, total drain output did not correlate with the need for postoperative blood transfusion $(p=0.22)$, or the total volume of blood transfused perioperatively ( $p=0.06)$ [35]. Blank et al. also analysed AIS patients with similar results [24]. Nevertheless, the group with drains received more postoperative autologous blood transfusions ( 0.88 vs. 0.5 units), but the difference was not statistically significant ( $p=0.2131$ ). Even the localisation (subcutaneous closed-suction drainage versus conventional closed-suction drainage) of the drain made no difference concerning transfusion requirements $(31.2 \%$ vs. $45.6 \%, p=0.133)$ in this collective of patients [16].

In other spinal surgeries the result were different. Diab et al. concluded that, after posterior spinal fusion surgery for other types of indications, patients with drains received more postoperative transfusions compared to those without (43\% vs. $22 \%, p<0.001$ ) [33]. Comparable with the results of Walid et al., the need for allogeneic blood transfusion was statistically more common in the drainage group $(23.9 \%$ vs. $6.8 \%, p=0.000)$ [34].

Gubin et al. demonstrated a significantly higher number of patients requiring blood transfusion after posterior spinal surgery involving more than one motion segment in the cohort with drains than those without (10\% vs. $0.1 \%)$ [27].

\section{Impact on costs and hospital stay}

Four investigations dealt with the economic impact dependent on length of stay and cost depending on the use of drains [18, 26, 34, 37].

None of the investigations found a longer length of stay or increased costs in the no-drain collective [26, 34, 37]. On the contrary, one investigation by Adogwa et al. on patients treated with spinal decompression and fusion found a significantly longer length of hospital stay for the drain-use cohort compared to the no-drain cohort (5.0 vs. 2.8 days, $p<0.0001$ ) [18]. Nevertheless, with a focus on lateral procedures involving three or more levels, the use of drains proved beneficial with a shorter length of stay and hospital charges compared to those without drains [34].

\section{Discussion}

The routine use of closed-suction drainage in thoracolumbar spinal surgery remains controversial. Wound drainage is placed with the intention of reducing postoperative complications. Many factors influence a surgeon's decision for or against drainage, mostly experience-based rather than evidence-based. After a thorough search, we evaluated 25 studies including 8579 participants for this systematic review. Only eight studies were prospective and/ or randomised. Thus, the conclusions should be considered cautiously.

Negative suction drainage leads to a significantly higher volume of drainage fluid in comparison with intermittent, overflow or subfascial drainages, respectively [34]. Whether the use of drainage triggers the need for blood transfusion or not is unclear. While studies with AIS patients did not show any elevated need for transfusion, the rate for blood transfusion was higher with regard to instrumented spinal surgery for other pathologies [16, 18, 24, 27, 32-36]. The reasons for these divergent results are certainly based on the different patient collectives. In particular, in the group of patients treated for idiopathic scoliosis, the predominantly younger age with a consecutively lower indication for transfusion could be responsible for this observation. Despite all the differences, drain volume alone should not be used as a trigger for recommending a postoperative blood transfusion [35].

A long duration of drainage treatment may cause a SSI but the time threshold remains unclear [34]. The current literature suggests that drainage duration well above $72 \mathrm{~h}$ may be associated with a higher incidence of SSIs [34]. Accompanying antibiotic treatment cannot decrease the incidence of SSIs and seems to be needless [34]. Routine drain tip cultures have not proven useful to predict SSI [34]. The impact of drainage on wound healing remains under debate. However, two prospective, randomised studies indicated that drains may not have an impact on wound healing [34].

Regarding postoperative fever, there are few studies with partially contradictory results. Wound drainage may increase a patient's temperature postoperatively. However, the elevated temperature seems not to be associated with any clinical sequelae [34]. Regarding postoperative haematomas, no evidence exists that wound drainage can prevent their formation [34]. The same is true regarding the influence of wound drainage on the prevention of neurological complications [34]. No studies exist regarding the rate of reoperations depending on the use of drainage. Hospital stay length and related costs may be elevated in patients with drainage but seems to depend on surgery type [18, 26, 34, 37].

This systematic review suffers from several limitations. The majority of included studies were retrospective case series (levels of evidence III and IV). There was no homogeneity. Most of the investigations were observational studies. Only papers published in English and German were included. Apart from three investigations, all studies included patients undergoing elective surgery. The selected inclusion criteria may not be comprehensive enough to reveal all published studies on the topic. The patient populations, spinal disorders and performed surgeries of the 
included studies differ considerably. Hence, inconsistent and to some extent controversial results were found.

\section{Conclusions}

Regarding the existing literature, the use of closed-suction drainage in elective thoracolumbar spinal surgery is not associated with any proven benefit for patients and does not appear to decrease postoperative complications. Negative pressure suction drainage increases drain volumes and may lead to a higher rate of blood transfusion. Drainage duration over $72 \mathrm{~h}$ can increase the risk of SSI.

In summary, the current literature does not justify the use of drainage in every patient. Therefore, the application should always be critically questioned. Further high-quality studies are necessary to reveal indications where drainage is justified.

Funding Open Access funding enabled and organized by Projekt DEAL.

\section{Declarations}

Conflict of interest The authors have no conflicts of interest to declare that are relevant to the content of this article.

Open Access This article is licensed under a Creative Commons Attribution 4.0 International License, which permits use, sharing, adaptation, distribution and reproduction in any medium or format, as long as you give appropriate credit to the original author(s) and the source, provide a link to the Creative Commons licence, and indicate if changes were made. The images or other third party material in this article are included in the article's Creative Commons licence, unless indicated otherwise in a credit line to the material. If material is not included in the article's Creative Commons licence and your intended use is not permitted by statutory regulation or exceeds the permitted use, you will need to obtain permission directly from the copyright holder. To view a copy of this licence, visit http://creativecommons.org/licenses/by/4.0/.

\section{References}

1. Di Lauro L, Poli R, Bortoluzzi M, Marini G (1982) Paresthesias after lumbar disc removal and their relationship to epidural hematoma. Report of two cases J Neurosurg 57(1):135-136. https://doi. org/10.3171/jns.1982.57.1.0135

2. Lawton MT, Porter RW, Heiserman JE, Jacobowitz R, Sonntag VK, Dickman CA (1995) Surgical management of spinal epidural hematoma: relationship between surgical timing and neurological outcome. J Neurosurg 83(1):1-7. https://doi.org/10.3171/jns.1995. 83.1.0001

3. Morse K, Weight M, Molinari R (2007) Extensive postoperative epidural hematoma after full anticoagulation: case report and review of the literature. J Spinal Cord Med 30(3):282-287. https:// doi.org/10.1080/10790268.2007.11753938
4. Takemoto RC, Lonner B, Andres T, Park J, Ricart-Hoffiz P, Bendo J, Goldstein J, Spivak J, Errico T (2015) Appropriateness of Twenty-four-Hour Antibiotic Prophylaxis After Spinal Surgery in Which a Drain Is Utilized: A Prospective Randomized Study. J Bone Joint Surg Am 97(12):979-986. https://doi.org/10.2106/ JBJS.L.00782

5. Parker MJ, Livingstone V, Clifton R, McKee A (2007) Closed suction surgical wound drainage after orthopaedic surgery. Cochrane Database Syst Rev 3:001825

6. Scuderi GJ, Brusovanik GV, Fitzhenry LN, Vaccaro AR (2005) Is wound drainage necessary after lumbar spinal fusion surgery? Med Sci Monit 11(2):64-66

7. Liu JM, Chen WZ, Fu BQ, Chen JW, Liu ZL, Huang SH (2016) The Use of Closed Suction Drainage in Lumbar Spinal Surgery: Is It Really Necessary? World Neurosurg 90:109-115. https://doi. org/10.1016/j.wneu.2016.02.091

8. Chen ZY, Gao Y, Chen W, Li X, Zhang YZ (2014) Is wound drainage necessary in hip arthroplasty? A meta-analysis of randomized controlled trials. Eur J Orthop Surg Traumatol 24(6):939-946. https://doi.org/10.1007/s00590-013-1284-0

9. Zhang QD, Guo WS, Zhang Q, Liu ZH, Cheng LM, Li ZR (2011) Comparison between closed suction drainage and nondrainage in total knee arthroplasty: a meta-analysis. J Arthroplasty 26(8):1265-1272. https://doi.org/10.1016/j.arth.2011.02.005

10. Payne DH, Fischgrund JS, Herkowitz HN, Barry RL, Kurz LT, Montgomery DM (1996) Efficacy of closed wound suction drainage after single-level lumbar laminectomy. J Spinal Disord 9(5):401-403

11. Kobayashi K, Imagama S, Ito Z, Ando K, Yagi H, Hida T, Ito K, Ishikawa Y, Tsushima M, Ishiguro N (2017) Is a Drain Tip Culture Required After Spinal Surgery? Clin Spine Surg 30(8):356-359. https://doi.org/10.1097/BSD.0000000000000326

12. Moher D, Liberati A, Tetzlaff J, Altman DG, Group P (2009) Preferred reporting items for systematic reviews and meta-analyses: the PRISMA statement. BMJ 339:b2535. https://doi.org/10.1136/ bmj.b2535

13. OloEW G (2011) The Oxford Levels of Evidence 2. Oxford Centre for Evidence-Based Medicine

14. Chen T, Chang H, Liu K, Shi M, Song C, Meng X (2020) Drainage after posterior single-level instrumented lumbar fusion: Natural pressure vs negative pressure. Medicine (Baltimore) 99(7):e19154. https://doi.org/10.1097/MD.0000000000019154

15. Hao QY, Liu CY, Fu CJ, Zhang XH, Tan MS (2016) Improved Intermittent-clamped Drainage in Lower Lumbar Internal Fixation: A Randomized Prospective Study. Chin Med J (Engl) 129(23):2804-2809. https://doi.org/10.4103/0366-6999.194639

16. Liang J, Qiu G, Chua S, Shen J (2013) Comparison between subcutaneous closed-suction drainage and conventional closedsuction drainage in adolescent idiopathic scoliosis patients undergoing posterior instrumented spinal fusion: a randomized control trial. J Spinal Disord Tech 26(5):256-259. https://doi.org/10.1097/ BSD.0b013e3182400897

17. Macki M, Uzosike A, Kerezoudis P, Bydon A, Bydon M, Gokaslan ZL (2017) Duration of indwelling drain following instrumented posterolateral fusion of the lumbar spine does not predict surgical site infection requiring reoperation. J Clin Neurosci 40:44-48. https://doi.org/10.1016/j.jocn.2016.12.008

18. Adogwa O, Elsamadicy AA, Sergesketter AR, Shammas RL, Vatsia S, Vuong VD, Khalid S, Cheng J, Bagley CA, Karikari IO (2018) Post-operative drain use in patients undergoing decompression and fusion: incidence of complications and symptomatic hematoma. J Spine Surg 4(2):220-226. https://doi.org/10.21037/ jss.2018.05.09

19. Ahn JS, Lee HJ, Park E, Park IY, Lee JW (2015) Suction Drain Tip Culture after Spine Surgery: Can It Predict a Surgical Site 
Infection? Asian Spine J 9(6):863-868. https://doi.org/10.4184/ asj.2015.9.6.863

20. Pennington Z, Lubelski D, Molina C, Westbroek EM, Ahmed AK, Sciubba DM (2019) Prolonged Post-surgical Drain Retention Increases Risk for Deep Wound Infection After Spine Surgery. World Neurosurg 130:e846-e853. https://doi.org/10.1016/j.wneu. 2019.07.013

21. Yamada T, Yoshii T, Egawa S, Takada R, Hirai T, Inose H, Kato T, Jinno T, Okawa A (2016) Drain Tip Culture is Not Prognostic for Surgical Site Infection in Spinal Surgery Under Prophylactic Use of Antibiotics. Spine 41(14):1179-1184

22. Urquhart JC, Collings D, Nutt L, Kuska L, Gurr KR, Siddiqi F, Rasoulinejad P, Fleming A, Collie J, Bailey CS (2019) The Effect of Prolonged Postoperative Antibiotic Administration on the Rate of Infection in Patients Undergoing Posterior Spinal Surgery Requiring a Closed-Suction Drain: A Randomized Controlled Trial. J Bone Joint Surg Am 101(19):1732-1740. https://doi.org/ 10.2106/JBJS.19.00009

23. Chen YC, Zhang L, Li EN, Ding LX, Zhang GA, Hou Y, Yuan W (2018) One or Two Drains for the Treatment of Surgical Site Infections After Lumbar Spine Surgery. World Neurosurg 116:e18-e25. https://doi.org/10.1016/j.wneu.2018.02.142

24. Blank J, Flynn JM, Bronson W, Ellman P, Pill SG, Lou JE, Dormans JP, Drummond DS, Ecker ML (2003) The use of postoperative subcutaneous closed suction drainage after posterior spinal fusion in adolescents with idiopathic scoliosis. J Spinal Disord Tech 16(6):508-512. https://doi.org/10.1097/00024720-20031 2000-00004

25. Alsiddiky A, Nisar KA, Alhuzaimi F, Albishi W, Alnuaim B, Albarrag M, Bakarman K, Meo SA (2013) Wound healing without drains in posterior spinal fusion in idiopathic scoliosis. J Coll Physicians Surg Pak 23(8):558-561

26. Ovadia D, Drexler M, Kramer M, Herman A, Lebel DE (2019) Closed Wound Subfascial Suction Drainage in Posterior Fusion Surgery for Adolescent Idiopathic Scoliosis: A Prospective Randomized Control Study. Spine 44(6):377-383

27. Gubin AV, Prudnikova OG, Subramanyam KN, Burtsev AV, Khomchenkov MV, Mundargi AV (2019) Role of closed drain after multi-level posterior spinal surgery in adults: a randomised open-label superiority trial. Eur Spine J 28(1):146-154. https:// doi.org/10.1007/s00586-018-5791-x

28. Li Z, Liu P, Zhang C, Xu G, Zhang Y, Chang Y, Liu M, Hou S (2019) Incidence, Prevalence, and Analysis of Risk Factors for Surgical Site Infection After Lumbar Fusion Surgery: $>/=2$-Year Follow-Up Retrospective Study. World Neurosurg 131:e460-e467. https://doi.org/10.1016/j.wneu.2019.07.207
29. Lai Q, Song Q, Guo R, Bi H, Liu X, Yu X, Zhu J, Dai M, Zhang B (2017) Risk factors for acute surgical site infections after lumbar surgery: a retrospective study. J Orthop Surg Res 12(1):116. https://doi.org/10.1186/s13018-017-0612-1

30. Liu JM, Deng HL, Chen XY, Zhou Y, Yang D, Duan MS, Huang SH, Liu ZL (2018) Risk Factors for Surgical Site Infection After Posterior Lumbar Spinal Surgery. Spine 43(10):732-737

31. Kanayama M, Oha F, Togawa D, Shigenobu K, Hashimoto T (2010) Is closed-suction drainage necessary for single-level lumbar decompression?: review of 560 cases. Clin Orthop Relat Res 468(10):2690-2694. https://doi.org/10.1007/s11999-010-1235-6

32. Kochai A, Erkorkmaz U (2019) The role of drains in adolescent idiopathic scoliosis surgery: Is it necessary? Medicine (Baltimore) 98(51):e18061. https://doi.org/10.1097/MD.0000000000018061

33. Diab M, Smucny M, Dormans JP, Erickson MA, Ibrahim K, Lenke LG, Sucato DJ, Sanders JO (2012) Use and outcomes of wound drain in spinal fusion for adolescent idiopathic scoliosis. Spine 37(11):966-973

34. Walid MS, Abbara M, Tolaymat A, Davis JR, Waits KD, Robinson JS 3rd, Robinson JS Jr (2012) The role of drains in lumbar spine fusion. World Neurosurg 77(3-4):564-568. https://doi.org/ 10.1016/j.wneu.2011.05.058

35. Choi DE, Vives MJ, Shah H, Zhao C, Patel A, Sabharwal S (2019) Do postoperative drain volumes correlate with intraoperative blood loss and postoperative transfusion requirements in posterior spinal fusion for adolescent idiopathic scoliosis? J Pediatr Orthop B 28(4):368-373. https://doi.org/10.1097/BPB.00000 00000000568

36. Adogwa O, Khalid SI, Elsamadicy AA, Voung VD, Lilly DT, Desai SA, Sergesketter AR, Cheng J, Karikari IO (2018) The use of subfascial drains after multi-level anterior cervical discectomy and fusion: does the data support its use? J Spine Surg 4(2):227232. https://doi.org/10.21037/jss.2018.05.10

37. Hung PI, Chang MC, Chou PH, Lin HH, Wang ST, Liu CL (2017) Is a drain tube necessary for minimally invasive lumbar spine fusion surgery? Eur Spine J 26(3):733-737. https://doi.org/10. 1007/s00586-016-4672-4

Publisher's Note Springer Nature remains neutral with regard to jurisdictional claims in published maps and institutional affiliations. 